\title{
A dysfunctional blood-brain barrier and cerebral small vessel disease
}

Atticus H. Hainsworth, $\mathrm{PhD}$

Mark J. Fisher, MD

Correspondence to Dr. Hainsworth:

ahainsworth@sgul.ac.uk and Dr. Fisher:

mfisher@uci.edu

Neurology ${ }^{\circledR}$ 2017;88:420-421

See page 426
In molecular terms, the blood-brain barrier (BBB) indicates tight junction complexes between adjacent endothelial cells that, combined with endothelial transporters (both influx and efflux), make brain endothelia different from other endothelia. While highly advantageous as a protective barrier against cerebrovascular permeability, shielding sensitive brain elements from blood-borne agents, it also poses a problem for those who seek to develop brain therapeutics such as designer antibodies targeted to amyloid deposits or tau protein derivatives.

Recent evidence from human studies indicates variation in barrier efficacy among patient populations. In this issue of Neurology ${ }^{\circledR}$, Zhang et al. ${ }^{1}$ used quantitative MRI measures (dynamic contrast-enhanced [DCE]MRI) to study cerebrovascular barrier function. They studied 116 older people, either with clinical and MRI evidence of cerebral small vessel disease or with no overt cerebrovascular disease. They used DCE-MRI to follow the time course of injected gadolinium-based contrast agent in each voxel of a brain MRI scan. They then used a standard analysis to derive 2 measures related to BBB function. These are the rate constant for gadolinium $(\mathrm{Gd})$ transfer from blood to brain (variously called $\mathrm{K}_{\mathrm{i}}$ or $\mathrm{K}_{\text {trans }}$ ) and the extent of brain tissue containing contrast agent $\left(\mathrm{v}_{\mathrm{L}}\right.$, the so-called fractional volume of leaky brain tissue), each derived for areas of white matter hyperintensity, for nonhyperintense white matter, for cortical gray matter, and for deep gray nuclei. They observed modest permeability, reflected in the $\mathrm{K}_{\mathrm{i}}$ and $\mathrm{v}_{\mathrm{L}}$ values, in all brain regions (highest in cortical gray matter) and across both participant groups. They found that older people with small vessel disease had a greater fraction of Gdpositive tissue (observed as greater $\mathrm{v}_{\mathrm{L}}$ ) relative to control individuals without small vessel disease. ${ }^{1}$ This held true within white matter hyperintensities, neighboring nonhyperintense white matter, and cortical gray matter (1.2- to 1.4-fold difference, depending on the tissue type). This picture agrees—broadly_with previous studies of older people with manifestations of small vessel disease (lacunar stroke, white matter hyperintensities, or vascular cognitive impairment). ${ }^{2-4}$
It may be a mistake to assume that a dysfunctional cerebrovascular barrier indicates breach of the tight junctions between endothelial cells. For many years, we have known about the trafficking of plasma substrates across the endothelial cytoplasm, known as transcytosis. Traditional emphasis on efflux transporters, which limit CNS concentration of penetrating drugs, has shifted to include analyses of influx mechanisms capable of enhancing drug delivery. ${ }^{5}$ Interest has recently increased with discovery of $\mathrm{Mfsd} 2 \mathrm{a}$, a $60 \mathrm{KDa}$ transmembrane protein greatly enriched in brain endothelium (>70-fold relative to peripheral endothelia). ${ }^{6}$ Mice genetically engineered to have no $M f s d 2 a$ gene exhibit increased BBB permeability due to increased transcytosis in brain endothelia. ${ }^{6}$ This pathway may be an entry route for therapeutic antibodies recently shown to reduce brain amyloid load. ${ }^{7}$ The current study ${ }^{1}$ leaves unclear the relative roles of paracellular vs transcellular barrier dysfunction.

Then again, is the demonstrated dysfunctional cerebrovascular barrier really an indication of endothelial disturbances at all? The ongoing assumption is that the barrier changes occur at the capillary level, but this remains an assumption. Arterioles, the vessels immediately upstream from capillaries, have layers of smooth muscle cells that normally provide barrier protection in addition to other arteriolar constituents. But small vessel disease, either hereditary or sporadic, is typically characterized by substantial loss of smooth muscle cells. ${ }^{8}$ Given that smooth muscle cells of arterioles may be no more than one layer thick, ${ }^{9}$ some arterioles in small vessel disease could contribute to loss of cerebrovascular barrier protection, a subject for further investigation.

The nature of the relationship between a dysfunctional cerebrovascular barrier and the pathogenesis of cerebral small vessel disease remains the critical question. The possible relationships, especially cause vs consequence, are complex and largely speculative at this point. Any unified model or fully developed theory linking white matter hyperintensities, microinfarcts, and cerebral microbleeds ${ }^{10}$ will need to incorporate conceptually a dysfunctional BBB.

From the Molecular and Clinical Sciences Research Institute (A.H.H.), St Georges University of London; Neurology (A.H.H.), St George's University Hospitals NHS Foundation Trust, London, UK; and Departments of Neurology, Anatomy \& Neurobiology, and Pathology \& Laboratory Medicine (M.J.F.), University of California, Irvine.

Go to Neurology.org for full disclosures. Funding information and disclosures deemed relevant by the authors, if any, are provided at the end of the editorial. 


\section{STUDY FUNDING}

No targeted funding reported.

\section{DISCLOSURE}

Work in Dr. Hainsworth's laboratory receives financial support from Alzheimer's Society (UK) (PG146/151), Alzheimer's Drug Discovery Foundation (Grant Ref 20140901), and Alzheimer's Research UK (PPG2014A-8). Dr. Hainsworth acknowledges honoraria from Eli Lilly, Dementia Discovery Fund, and National Institute on Aging. Dr. Hainsworth served on the Vascular Contributions to Cognitive Impairment and Dementia panel for the Alzheimer's Disease Related Dementias Summit, NIH, March 2016. Dr. Fisher is supported by NIH RO1 NS20989 (Dr. Fisher). Dr. Fisher also has research grants from Otsuka Pharmaceutical Company and Boehringer-Ingelheim, and serves on clinical event committees for Covidien. Go to Neurology.org for full disclosures.

\section{REFERENCES}

1. Zhang CE, Wong SM, van de Haar HJ, et al. Blood-brain barrier leakage is more widespread in patients with cerebral small vessel disease. Neurology 2017;88:426-432.

2. Huisa BN, Caprihan A, Thompson J, Prestopnik J, Qualls CR, Rosenberg GA. Long-term blood-brain barrier permeability changes in Binswanger disease. Stroke 2015;46:2413-2418.

3. Topakian R, Barrick TR, Howe FA, Markus HS. Bloodbrain barrier permeability is increased in normal-appearing white matter in patients with lacunar stroke and leucoaraiosis. J Neurol Neurosurg Psychiatry 2010;81:192-197.

4. Wardlaw JM, Doubal F, Armitage P, et al. Lacunar stroke is associated with diffuse blood-brain barrier dysfunction. Ann Neurol 2009;65:194-202.

5. Sanchez-Covarrubias L, Slosky LM, Thompson BJ, Davis TP, Ronaldson PT. Transporters at CNS barrier sites: obstacles or opportunities for drug delivery? Curr Pharm Des 2014;20:1422-1449.

6. Ben-Zvi A, Lacoste B, Kur E, et al. Mfsd2a is critical for the formation and function of the blood-brain barrier. Nature 2014;509:507-511.

7. Sevigny J, Chiao P, Bussiere T, et al. The antibody aducanumab reduces Abeta plaques in Alzheimer's disease. Nature 2016;537:50-56.

8. Craggs LJL, Yamamoto Y, Deramecourt V, Kalaria RN. Microvascular pathology and morphometrics of sporadic and hereditary small vessel diseases of the brain. Brain Pathol 2014; 24:495-509.

9. Martinez-Lemus LA. The dynamic structure of arterioles. Basic Clin Pharmacol Toxicol 2011;110:5-11.

10. Fisher M. Cerebral microbleeds and thrombolysis: clinical consequences and mechanistic implications. JAMA Neurol 2016;73:632-635. 